\title{
Analysis and investigation of a differential interferometer based on a polymer optical bimodal waveguide
}

\author{
K. Gut, ${ }^{*}$ T. Herzog \\ Department of Optoelectronics, Silesian University of Technology, Akademicka 2A, 44-100 Gliwice
}

Received May 20, 2015; accepted June 01, 2015; published June 30, 2015

\begin{abstract}
The paper presents analysis of a differential interferometer based on a polymer bimodal optical waveguide. For the structure of $\mathrm{SiO}_{2}$ / $\mathrm{SU} 8 / \mathrm{H}_{2} \mathrm{O}$ the waveguide thickness that corresponds to the maximum sensitivity has been determined. The paper presents the possibility of detecting the phase difference between the modes through registrations of only a part of energy propagating in the waveguide. The experimentally determined values of attenuation for the guided modes $\mathrm{TE}_{0}$ and $\mathrm{TE}_{1}$ are presented, too.
\end{abstract}

In recent years, planar waveguide optical interferometers have been gaining attention in biosensors applications [1]. Particularly noteworthy are differential interferometers using a two-mode optical waveguide [25], which in some cases provide greater sensitivity than single-mode waveguides [6]. They operate utilizing the interference of two modes of different orders in the exemplary types of structures: spiral-type waveguides [7], silicon nanowire ridge waveguides (SNRW) [8], composite bimodal waveguides [9]. Modes propagating in a waveguide interfere with each other and the light intensity distribution at the output of the structure depends on the phase difference between the modes. In the papers $[10,11]$, the authors have shown experimentally (probably for the first time in the literature of the problem) the effect of a varying refractive index cover on the intensity distribution in modes at the output.

The three-layer planar structure was chosen as a model for numerical analysis. The analysed structure is presented in Fig.1. The thicknesses of $\mathrm{SiO}_{2}$ and $\mathrm{H}_{2} \mathrm{O}$ layers were $10 \mu \mathrm{m}$.

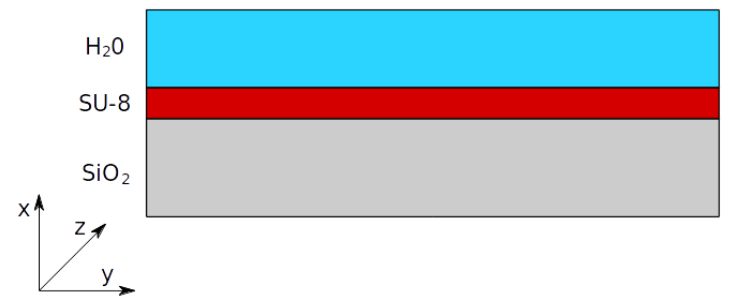

Fig.1. Configuration of layered optical structure.

The influence of the SU-8 layer thickness on modes propagation was subject to numerical analysis. All simulations were performed with the assumption that the wavelength of the laser is $\lambda=633 \mathrm{~nm}$. The refractive indexes of $\mathrm{SiO}_{2}$ and $\mathrm{SU}-8$ were taken from our ellipsometric measurements and equal $n_{s}=1.4660$ and $\mathrm{n}_{\mathrm{f}}=1.6170$, respectively. The refractive index for $\mathrm{H}_{2} \mathrm{O}$ was taken from Ref. [12] and its value is assumed as $\mathrm{n}_{\mathrm{c}}=1.3317$. By solving numerically the guided mode dispersion equation [13] effective refractive index for a given thickness of the polymer layer can be found. Figure 2 presents the effective refractive index $(N)$ as a function of SU-8 thickness computed using the OptiWave Software. In this paper we analyzed only the structure that supports the $\mathrm{TE}_{0}$ and $\mathrm{TE}_{1}$ modes.

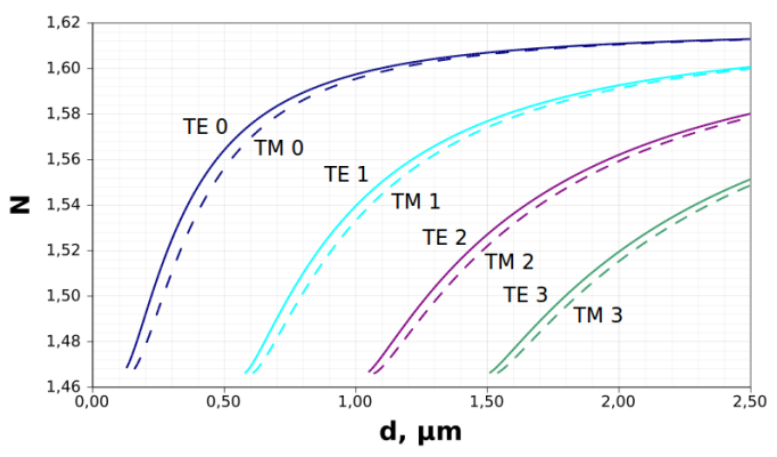

Fig. 2. Effective refractive indices as a function of SU-8 layer thickness.

In the case of an interferential system, the mode sensitivity $S_{i}\left(n_{c}\right)$ is a very important parameter. It describes the changes of the effective refractive index $\Delta N$ due to changes in the refractive index of the cover $\Delta n_{c}$

$$
S_{i}\left(n_{c}\right)=\frac{\Delta N}{\Delta n_{c}}
$$

Knowing the effective refractive indices, the mode sensitivity can be determined. The sensitivity $S_{i}\left(n_{c}\right)$ for TE modes is determined by the formula [14]:

$$
S_{i}\left(n_{c}\right)=\left(\frac{n_{c}}{N}\right)\left(\frac{n_{f}^{2}-N^{2}}{n_{f}^{2}-n_{c}^{2}}\right)\left(\frac{\Delta z_{c}}{d_{e f f}}\right)
$$

Where, $d_{\text {eff }}$ is the effective thickness of mode penetration and can be defined as:

$$
d_{\text {eff }}=d_{f}+\Delta z_{c}+\Delta z_{s}
$$

In Eq. (3) $d_{f}$ is the SU-8 thickness, $\Delta \mathrm{z}_{\mathrm{c}}$ is the depth of mode penetration into the cover $\left(\mathrm{H}_{2} \mathrm{O}\right)$ and $\Delta \mathrm{z}_{\mathrm{s}}$ is the depth of mode penetration into the substrate $\left(\mathrm{SiO}_{2}\right)$.

\footnotetext{
*E-mail: kazimierz.gut@polsl.pl
} 


$$
\Delta z_{j=s \vee c}=\frac{\lambda}{2 \pi} \frac{1}{\sqrt{N^{2}-n_{j}^{2}}}
$$

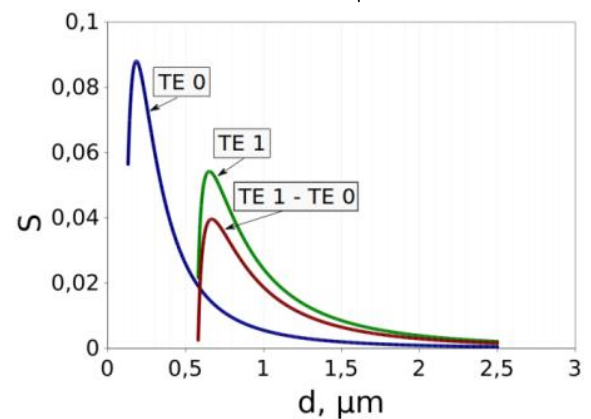

Fig. 3. Homogenous sensitivity of the structure as a function of SU-8 layer thickness.

Two modes can be excited in the analyzed waveguide. A change in the refractive index of the cover causes changes in the effective refractive indices of the guided modes. The sensitivity of the differential interferometer $S_{d}\left(n_{c}\right)$ can be determined as differences in the mode sensitivity $S_{\mathrm{TE} 1}\left(n_{c}\right)$ and $S_{T E O}\left(n_{c}\right)$ of the guided modes. The sensitivity of the differential interferometer is presented in Fig. 3. The maximum sensitivity amounted to 0.0396 at the $0.67 \mu \mathrm{m}$ thickness of an SU-8 layer. This thickness was used in further analysis of light distribution in the structure.

The numerical analysis of electric field distributions of $\mathrm{TE}_{1}$ and $\mathrm{TE}_{0}$ modes was obtained by means of the OptiWave Software using the Transfer Matrix Method (TMM). Light intensity distribution was obtained by using Eq. (5). The energy in each mode was calculated using Eq. (6) and normalized to 1 .

$$
\begin{gathered}
I(x) \square E^{2}(x) \\
\int_{0}^{x} E^{2}(x) d x \approx \sum_{0}^{x}\left(E^{2}(x) \cdot \Delta x\right)
\end{gathered}
$$

To obtain light intensity distribution, when intermodal interference occurs as a function of phase difference $\phi$ between modes, electric fields in each point were treated like phasors and were summed. The light intensity at a given point on the $x$-axis is proportional to the square of the sum of the phasors corresponding to the $\mathrm{TE}_{0}, \mathrm{TE}_{1}$ modes and the phase difference $\phi$ (Fig.4).

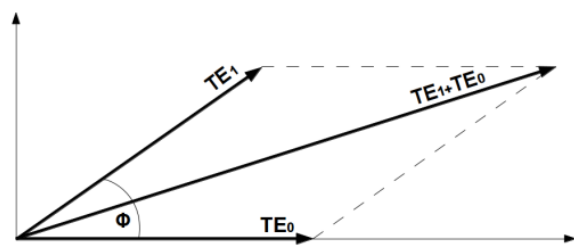

Fig. 4. Example of phasor sum.
The obtained light distribution in the structure for phase difference of $0^{\circ}, 45^{\circ}$ and $90^{\circ}$ is presented in Figs. 5-7.

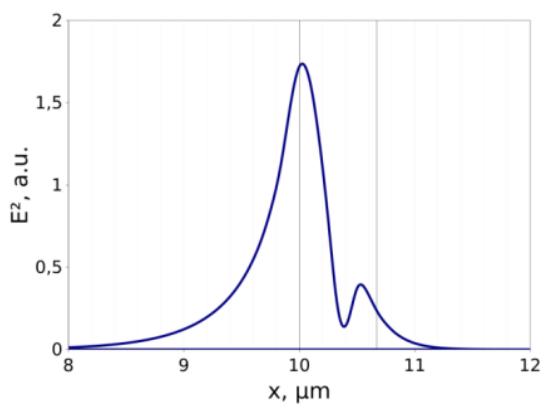

Fig. 5. Light intensity distribution with phase difference $0^{\circ}$.

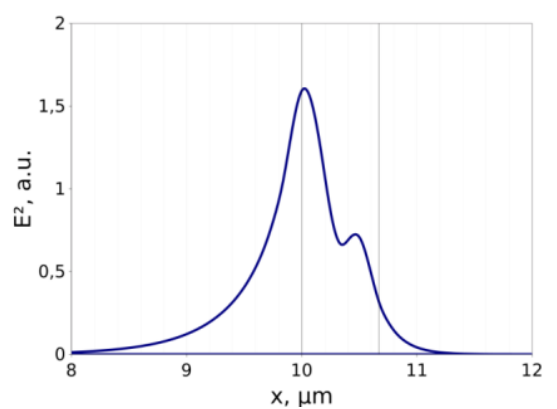

Fig. 6. Light intensity distribution with phase difference $45^{\circ}$.

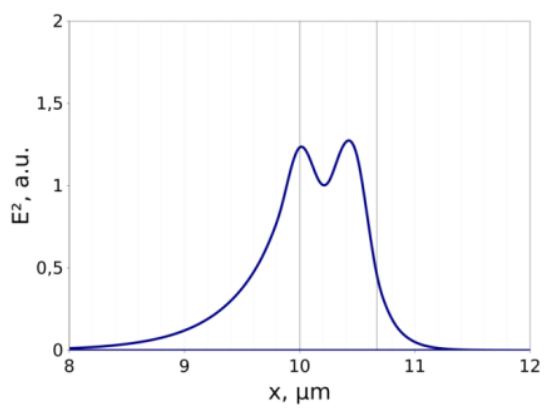

Fig. 7. Light intensity distribution with phase difference $90^{\circ}$.

The intensity distribution of the light at the end of the waveguide is a function of the phase difference between the carried modes. Our idea of phase difference detection was based on utilizing both the detector and the absorbent at the waveguide output (Fig 8.)

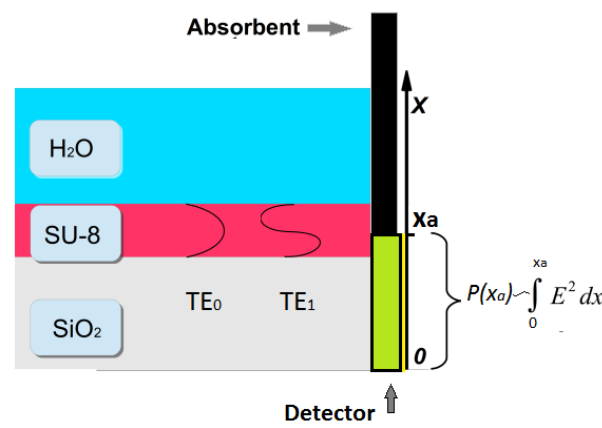

Fig. 8. Idea of the phase difference detection. 
The detector captures the part of running light power in the waveguide while the remaining part is absorbed. The border between the detector and the absorber is determined by $x_{a}$ coordinate. The power delivery to the detector is described by Eq. (7) and is a function of the phase difference between the modes and depends on $x_{a}$.

$$
P\left(\phi, x_{a}\right) \square \int_{0}^{x_{a}} E^{2}(x) d x .
$$

For a given position of the point $x_{a}, P(x, \phi)$ is only dependent on $\phi$. To determine the optimal position of the $x_{a}$, the $r\left(x_{a}\right)$ parameter that describes the maximum relative change of the recorded signal has been defined as

$$
r\left(x_{a}\right)=\frac{P_{\max }\left(x_{a}\right)-P_{\min }\left(x_{a}\right)}{P_{\max }\left(x_{a}\right)}
$$

where $P_{\max }\left(x_{a}\right)$ and $P_{\min }\left(x_{a}\right)$ are the maximum and minimum values of the light power for phase changes $\phi$ from 0 to $2 \pi$, for a fixed value of $x_{a}$.

The dependence of the value of the $r\left(x_{a}\right)$ parameter on the position of the point $x_{a}$ is shown in Fig. 9. The relative change of power has a maximum value of 0.54 at $x_{a}=10.09 \mu \mathrm{m}$.

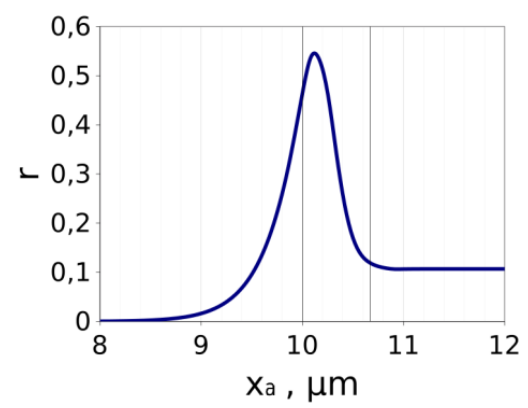

Fig. 9. Dependence of the parameter $\mathrm{r}$ on the position of the point $x_{a}$.

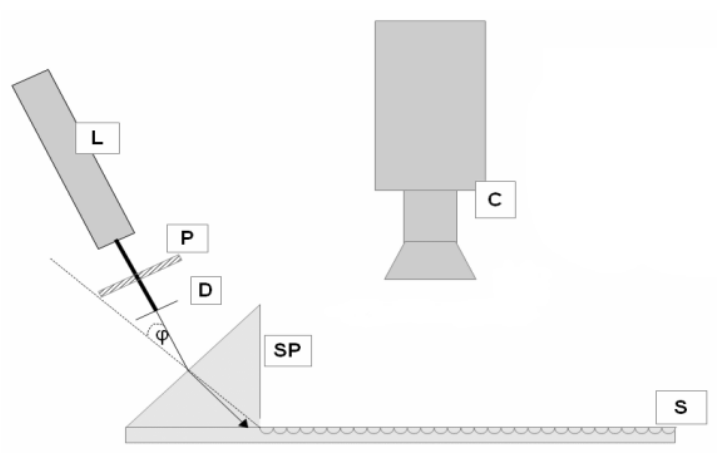

Fig. 10. Experimental setup [15].

All analyses were made with an assumption that energies in $\mathrm{TE}_{1}$ and $\mathrm{TE}_{0}$ modes are equal.

We have made a preliminary test to evaluate the attenuation in $\mathrm{TE}_{1}$ and $\mathrm{TE}_{0}$ modes. The experimental setup for using scattered light to measure attenuation is presented in Fig. 10 [15]. The experimental setup consists of: a laser (L), camera (C), polariser (P), diaphragm (D) and prism coupler (SP). The source of light, i.e. a semiconducting edge laser, emitting at a wavelength of $\lambda=0.663 \mu \mathrm{m}$, was mounted on a rotary arm, which warranted the introduction of the beam at an adequate angle corresponding to the $\mathrm{TE}_{0}$ or $\mathrm{TE}_{1}$. The image was detected by means of a black-and-white camera GKB(NO. CB23803SA 1/3 IR BWCCD, the number of pixels: $768 \times 494)$. We received an image with a resolution of 8 bits. It is of extreme importance that the camera be equipped with an adequate CCD transducer with linear sensitivity characteristics in the given range of wavelength in which the investigations are being run [16]. Measurements attenuation by the detection of scattered light in planar structures is based on the relation in which the intensity of scattered light is proportional to that of propagated light [17]. A detailed description of the measurement system and method for determining the attenuation can be found in [15] and [16]. The values of attenuation of modes obtained in this setup are $7.5 \mathrm{~dB} / \mathrm{cm}$ for $\mathrm{TE}_{0}$ and $3.6 \mathrm{~dB} / \mathrm{cm}$ for $\mathrm{TE}_{1}$.

This is a preliminary examination of the polymer (SU-8) waveguide structure on a $2 \mu \mathrm{m} \mathrm{SiO}_{2}$ substrate on $\mathrm{Si}$. The obtained results have shown that such waveguides can find practical applications in photonic sensors.

The investigations of structures based on the SU-8 polymer will be continued.

This work was supported by the Polish National Science Centre (NCN) under the grant 2012/07/B/ST7/01471

\section{References}

[1] P. Kozma et al., Biosens. Bioelectron. 58, 287 (2014).

[2] S. Dante et al., Laser Photonics Rev. 9, 248 (2015).

[3] M. Estevez et al., Laser Photonics Rev. 6, 463 (2012).

[4] R. Bruck, R. Hainberger, Opt. Expr. 22, 32344 (2014).

[5] K.E. Zinoviev et al., Opt. Expr. 29, 1926 (2011).

[6] K. Gut, Opt.Appl. 42, 407 (2012).

[7] Q. Liu et al., Opt. Expr. 22, 27910 (2014).

[8] R. Gamal et al., J. Opt. 17, 045802 (2015).

[9] K. Gut, Acta Physic Pol. A 124, 602 (2013).

[10] W.T. Wójcik et al., Proc. SPIE 4239, 192 (2000).

[11] K. Gut et al., Opt. Appl. 29, 101 (1999).

[12] M. Daimon, A. Masumura, Appl. Optics 46, 3811 (2007).

[13] A. Boudrioua, Photonic waveguides (Hoboken, John Wiley \& Sons, Inc. 2009).

[14] K. Tiefenthaler, W. Lukosz, J. Opt. Soc. Am. B 6, 209 (1989).

[15] K. Gut, T. Herzog, Elektronika 11, 53 (2012).

[16] K. Gut, K. Nowak, Eur. Phys. J.-Spec. Top. 154, 89 (2008).

[17] T.A. Strasser, M.C. Gupta, Appl. Opt. 31, 2041 (1992). 